\title{
Correlative Microscopy of Tumor Vasculature
}

\author{
P.J. Kempen,* B.R. Smith,** M.F. Kircher,*** S.S. Gambhir,** and R. Sinclair* \\ * Department of Materials Science and Engineering, Stanford University, 496 Lomita Mall, \\ Stanford, CA 94305-4034 \\ **Molecular Imaging Program at Stanford, Departments of Radiology \& Bioengineering, Stanford \\ University, Stanford CA 94305 \\ ***Department of Radiology, Memorial Sloan-Kettering Cancer Center, 1275 York Avenue A-288, \\ New York, NY 10065
}

As a tumor grows, the blood vessels inside the tumor must grow as well to continue feeding it. Owing to the accelerated growth of the blood vessels as well as the presence of mutations, the vessel does not grow perfectly and defects form along the vasculature[1]. This results in what is commonly termed leaky vasculature. This phenomenon has successfully been utilized in both the diagnosis and treatment of cancer[2,3]. The presence of defects allow the targeting agent, such as a nanoparticle, to extravasate and accumulate in the tumor either aiding in imaging for diagnosis or targeted drug delivery for treatment[2,3]. The goal of this work is to utilize correlative microscopy to locate and characterize the tumor vasculature as well as the extravasation of nanoparticles into the tumor.

Many of the tumor vasculature defects and nanoparticles are on the order of tens of nanometers, making them too small to be resolved by traditional light microscopy techniques. Scanning electron microscopy is well suited to image at this scale[1], however locating blood vessels using SEM is a difficult task. We can utilize light microscopy to locate blood vessels within the tumor and take correlative SEM images to analyze the vessel at higher resolution.

Two tumor models were analyzed in this work, a LS174T colon tumor and an Ingo4 brain tumor injected with gold-silica core-shell nanoparticles. The LS174T tumor was fixed with a solution of $0.5 \%$ glutaraldehyde $1 \%$ paraformaldehyde in $0.075 \mathrm{~mol} / \mathrm{L}$ sodium cacodylate and processed using standard histological methods[4]. The Ingo4 tumor was fast frozen in O.C.T. 4 and $10 \mu \mathrm{m}$ sections were cut from the LS174T and Ingo4 tumors respectively and stained using hematoxylin and eosin stain. This protocol stains red blood cells bright red, figure 1(B), allowing us to locate vessels. The sections were imaged using a Leica DM 2000 microscope and stitched together creating an image of the tumor, figure 1(A). The glass slides were then coated with a thin, 1nm, AuPd film to improve sample conductivity and imaged using a FEI Magellan XHR SEM. LS174T sections were imaged at $1 \mathrm{kV}$ with a probe current of $13 \mathrm{pA}$, to minimize sample damage and charging. The blood vessel, Figure 1(B), located in the light microscope was imaged again using the SEM as shown in Figure 1(C). From this image we can clearly identify red blood cells as well as the endothelial cells that comprise the blood vessel wall. Ingo4 sections were imaged at $15 \mathrm{kV}$ with a probe current of $50 \mathrm{pA}$ to optimize the backscattered electron signal from the gold core nanoparticles. Nanoparticle clusters, figures $2(\mathrm{~A}-\mathrm{C})$, were located in the tumor region of the tissue slice indicating the extravasation of the nanoparticles out of the vasculature.

Combining light and scanning electron microscopy creates a powerful tool to help locate and analyze areas of samples that would otherwise be too difficult and time consuming to image. Correlative microscopy provides an excellent tool to study tumor vasculature and extavasation effects. 
References

[1] H. Hashizume et al., American Journal of Pathology 156 (2000) 1363

[2] B.R. Smith et al., Nano Letters 8 (2008) 2599

[3] S.K. Sahoo and V. Labhasetwar, Drug Discovery Today 8 (2003) 1112

[4] L. Weiss, Cell and Tissue Biology: A textbook of Histology, 6th ed., Urban \& Schwarzenberg, Baltimore, 1988.

[5] This research is supported by the Center for Cancer Nanotechnology Excellence Focused on Therapy Response (CCNE-TR) grant NCI CCNE U54 CA119367 (SSG)

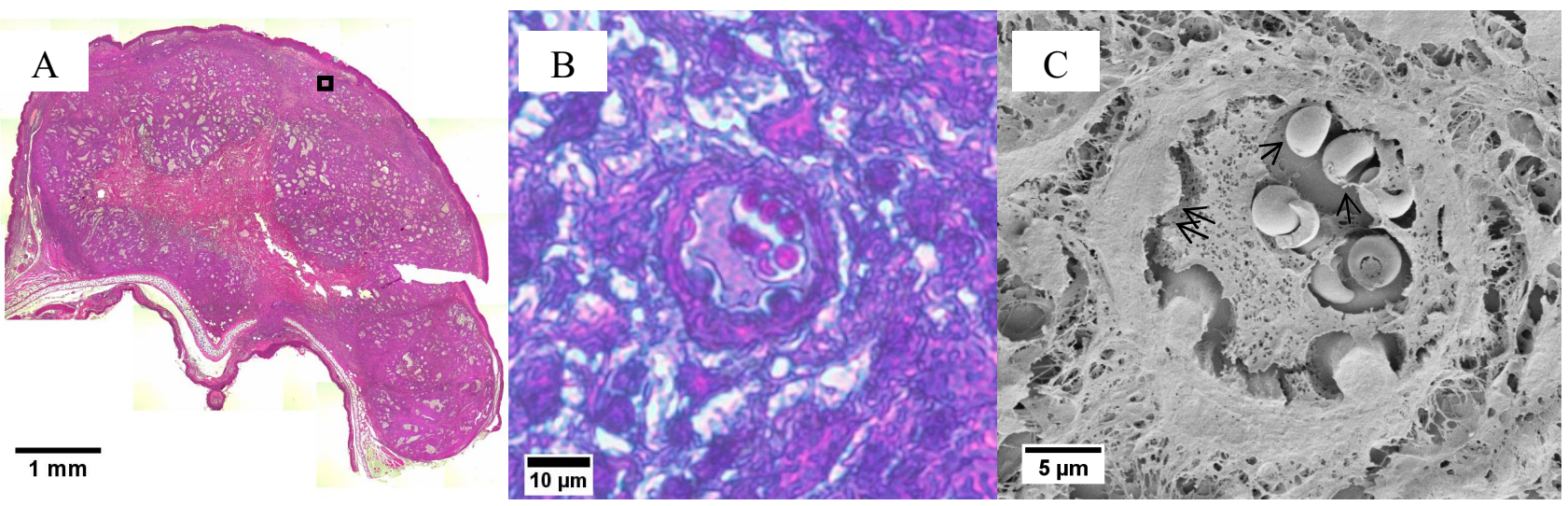

FIG. 1. (A) H\&E stained LS174T tumor section with box indicating the (B) magnified region showing a blood vessel with red blood cells. (C) Secondary electron image of the blood vessel shown in (B). Red blood cells are clearly visible, denoted with arrow, as well as endothelial cells that comprise the vessel wall, denoted double arrow.

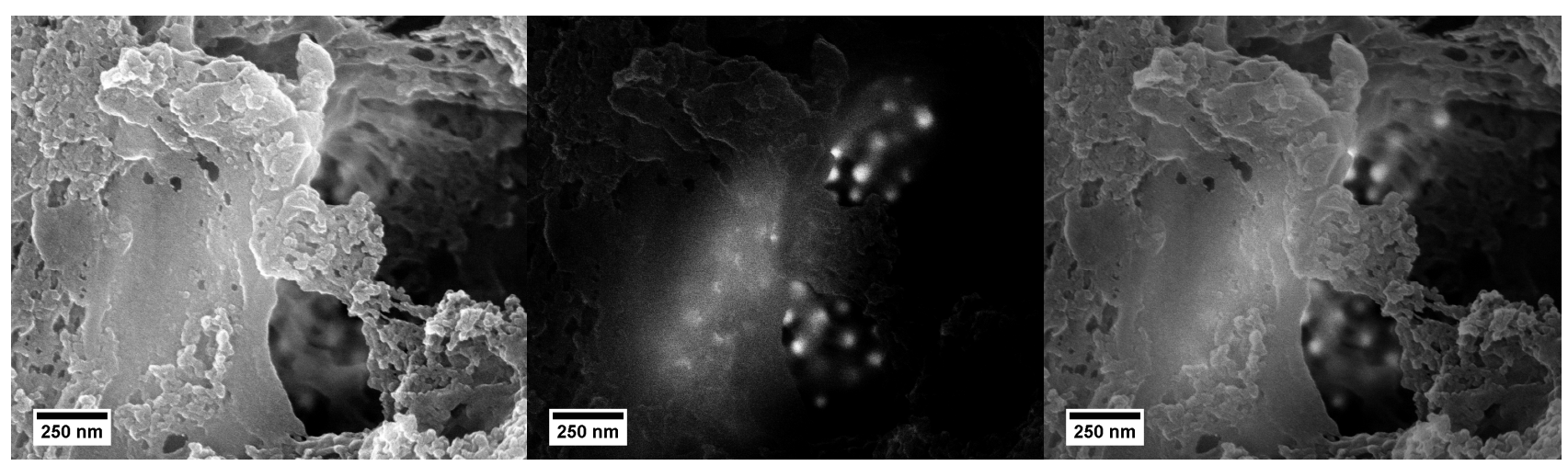

FIG. 2. (A) Secondary electron image showing to surface of the Ingo4 tumor tissue with nanoparticles inside it. (B) Backscattered electron image showing the gold core of the nanoparticles as bright white against a dark background from the same area. (C) 1:1 mixed secondary and backscattered electron image showing the nanoparticles under the surface of the tumor tissue section. 